\title{
The representation of nonstructural information in visual memory: Evidence from image combination
}

\author{
PETER WALKER and GRAHAM J. HITCH \\ Lancaster University, Lancaster, England \\ STEPHEN A. DEWHURST and HELEN E. WHITELEY \\ University of Central Lancashire, Preston, England \\ and \\ MARIA A. BRANDIMONTE \\ University of Trieste, Trieste, Italy
}

\begin{abstract}
Two experiments investigated the differential representation of the figure and ground of a picture in visual short-term and long-term memory. It is known (Hitch, Brandimonte, \& Walker, 1995) that subjects find it more difficult to combine mental images of two separately presented pictures in order to identify a novel form when the two pictures are incongruent in color (i.e., when a black-on-white line drawing has to be combined with a white-on-black drawing). In the present experiments, the figures were depicted in solid form to allow color congruity to be varied independently for figure and ground. Results showed a clear impairment in image combination when the to-be-combined figures were incongruent in color (black-on-gray and white-on-gray) but not when their grounds were incongruently colored (gray-on-black and gray-on-white). In this way, image combination was seen to be supported by a representation of the object depicted in the picture rather than by a literal representation of the picture itself (i.e., a pictorial code). In line with previous findings, the same representation was seen to support image combination based on short-term memory (Experiment 1) and long-term memory (Experiment 2), provided that in the latter case verbal recoding was precluded. When verbal recoding was allowed, image combination based on long-term memory was insensitive to color congruity, implying the involvement of a more abstract structural representation.
\end{abstract}

Although the distinction between short-term and longterm memory has been discussed most in relation to verbal memory, a similar distinction has been proposed for visual memory (Avons \& Phillips, 1980, 1987; Phillips, 1983). The fundamental distinction in both domains concerns the durability of representations, but there are other distinctions common to the two domains. For example, in contrast with long-term memory, both verbal and visual short-term memory are assumed to have limited capacity. According to one view, visual short-term memory can preserve information about a single item only, typically the most recent, and is further limited by the complexity of this item (Avons \& Phillips, 1980; Kikuchi, 1987; Phillips, 1983).

In addition, in each domain, controlled processes are thought to counteract the lability of the representations in short-term memory and to perform operations on them. In-

This research was supported by a grant from the University of Central Lancashire awarded to P.W., H.E.W., and G.J.H. S.A.D. is now at the Department of Psychology, Lancaster University. Correspondence should be addressed to P. Walker, Department of Psychology, Lancaster University, Lancaster LA1 4YF, England (e-mail: p.walker $(a$ lancaster.ac.uk). deed, short-term visual memory has been characterized as an active process of visualization that sustains a detailed representation of an item in order that it can, for example, be compared with a succeeding item (see Christie \& Phillips, 1979; Phillips \& Christie, 1977a, 1977b). In the context of visual imagery, Farah (1984) and Kosslyn (1980) have argued that the maintenance, inspection, and manipulation of visual images is mediated by operations on the contents of a limited capacity short-term visual buffer, which can be fed by a recent percept or an image derived from long-term memory.

Finally, in both domains it has been assumed that the durability of a memory representation is directly related to its level of abstractness-a view that is the essence of the levels of processing approach to memory (Craik \& Lockhart, 1972). In the visual domain, increased abstractness has been associated with enhanced durability and with invulnerability to the presentation of succeeding visual stimuli (Avons \& Phillips, 1987; Ellis, Allport, Humphreys, \& Collis, 1989). For example, Ellis et al. distinguished two types of representation involved in the recognition of pictures of objects. One type preserves information that is specific to the conditions under which the depicted object is viewed (e.g., lighting, angle of view, 
and viewing distance). The other type of representation is more abstract and preserves structural information independently of view-specific features (e.g., a description of the spatial arrangement of a sample of primitive volumetric forms, or geons, such as spheres, cylinders, and wedges; see Biederman, 1987). Using a picture matching task, Ellis et al. demonstrated that the first type of representation, though derived quickly, is less durable and more susceptible to interference from the presentation of additional visual information. This is consistent with the idea that long-term visual memory is associated with relatively more abstract representations than is shortterm visual memory. However, more recent evidence illustrates that long-term visual memory can draw on either type of representation depending on task demands. Biederman and Cooper $(1991,1992)$ and Cooper and Schacter (1992) have shown that long-term explicit recognition memory for pictures of objects draws on a view-specific representation, as revealed by a sensitivity to changes in the size, location, and left-right orientation of the depicted object. On the other hand, Biederman and Cooper $(1991,1992)$ and Biederman and Gerhardstein (1993) have examined long-term priming effects in the naming of pictured objects and observed a lack of sensitivity to nonstructural differences between the priming and primed objects, implying the involvement of a structural representation. Similarly, Cooper, Schacter, and their colleagues have demonstrated that priming in the object decision task is not diminished when the priming and primed objects differ in size or in leftright orientation (Cooper \& Schacter, 1992; Cooper, Schacter, Ballesteros, \& Moore, 1992; Schacter, Cooper, \& Delaney, 1990; Schacter, Cooper, Delaney, Peterson, \& Tharan, 1991). It would appear, therefore, that whereas short-term visual memory normally draws on view-specific representations, long-term memory can draw on either view-specific or view-independent representations, depending on task demands.

Further evidence for distinct forms of visual representation emerges from differential associations with the verbal memory system and with verbal processes more generally. It is now known that verbal recoding can influence performance on visual imagery tasks and does so differently according to whether the tasks draw on short-term or long-term visual memory (Brandimonte, Hitch, \& Bishop, 1992a, 1992b, 1992c; Hitch, Brandimonte, \& Walker, 1995). For example, Brandimonte et al. (1992b) and Hitch et al. explored an image combination task in which subjects were required to superimpose two visual images, a remembered image and a current image of a recent percept, in order to identify an emergent form. The emergent form could not be identified from either one of the component images alone, but instead required the images to be accurately superimposed (Figure 1). In a short-term memory condition, the to-be-combined visual stimuli were presented in immediate succession. In a long-term memory condition, there was a learning phase in which the set of first members of the pairs of to-be-combined stimuli were repeatedly pre- sented until the subject could visualize each one in turn. Then, in the testing phase, the second member of each pair was presented in turn and subjects had to combine each one with an image of its partner from the learned set. The impact of verbal recoding on image manipulation was assessed in two ways in these studies--by varying the nameability of the to-be-manipulated items and by requiring subjects to suppress articulation during the presentation and retention of nameable items. Whereas performance based on short-term memory was insensitive to verbal recoding, performance based on long-term memory was enhanced when verbal recoding was precluded. This is taken as evidence that short-term visual memory is supported by literal representations of visual stimuli independently of verbal processes. It is also taken as evidence that long-term visual memory is normally supported by representations that are more abstract, though still sufficiently close to the projected shape to support image combination, and whose use is contingent on verbal recoding. In addition, however, the results have been taken to imply that, when verbal recoding is precluded, long-term visual memory can also draw on a representation equivalent to that supporting short-term visual memory.

In the most recent of these studies, Hitch, Brandimonte, and Walker (1995) enquired about any qualitative differences in the nature of the two forms of representation supporting image combination. More specifically, they tested the idea that whereas short-term visual memory preserves the surface features of objects (and features that generally would be specific to the viewing conditions), long-term memory normally does not. To this end they manipulated a surface feature of the to-be-combined stimuli, namely their color (black/white). Whereas in the earlier image manipulation experiments the stimuli were always black line drawings on a white surface, in their experiment the stimuli were produced in two versions, as black-on-white and white-on-black pictures. In a condition in which the surface features of the to-be-combined stimuli were congruent, both stimuli appeared either as black-on-white or as white-on-black pictures. In a condition in which the surface features of the to-be-combined stimuli were incongruent, one picture was black-on-white and one was white-on-black. It was argued that if the representation supporting image combination preserves surface details, color incongruity should have a detrimental effect on performance. If, however, the representation does not incorporate such details, but is purely structural, color incongruity should have no effect. Hitch et al. studied image combination with nameable stimuli and found that performance in the short-term condition was sensitive to color congruity in the predicted way, whereas performance in the long-term condition was insensitive to color congruity. Furthermore, in agreement with earlier results concerning the impact of verbal recoding, when articulation was suppressed, performance in the longterm condition became sensitive to color congruity in just the same way as performance in the short-term condition. Once again, it was as if precluding verbal recoding 
induced a switch from relying on a view-independent structural description to relying on a representation equivalent to that associated with short-term memory. All this was interpreted by Hitch et al. as support for Kosslyn's (1980) view that whereas short-term visual memory is concerned solely with the visual appearance of things, long-term visual memory is concerned with both the visual appearance of things and their structure. Furthermore, they concluded that whereas visual short-term memory is independent of verbal processes, visual longterm memory interconnects with such processes and with verbal long-term memory.

Exactly how visual representations preserve view-specific information from a picture requires further exploration. For instance, view-independent structural representations are object based, but it is uncertain whether this is also the case for view-specific representation. It is possible, for example, that view-specific information is preserved through a literal representation of the visual stimulus (i.e., the picture) rather than through a more derived representation of the depicted object. In their investigation of priming effects in the identification of pictures of familiar objects, Warren and Morton (1982) claimed that repetition priming between identical pictures was mediated by a literal representation of the first picture. Similarly, with regard to memory for pictures of faces, a distinction has been drawn between a viewindependent structural code of a depicted face and a literal representation of the picture itself (Bruce \& Valentine, 1985; Roberts \& Bruce, 1989; Young, Hay, McWeeny, Flude, \& Ellis, 1985). Bruce and Young (1986) have called this literal representation the "pictorial code" and characterized it as a 2-D description incorporating details of the grain and flaws in a picture, as well as features relating to lighting, static pose, and facial expression. They have argued that the same code can be established for any visual pattern.

The present study focuses on the nature of the visual representation preserving surface color in image combination and the question of whether this is a representation of the picture itself or a representation of the object depicted in the picture. To decide between these two alternatives, the color congruity of the drawn object (the figure) was manipulated independently of the color congruity of the background picture surface (the ground). When a literal representation of the picture is used, both manipulations of congruity would be expected to influence image combination, since both figure and ground are integral aspects of the picture. In contrast, when a representation of the depicted object alone is used, the color congruity of the figure should influence image combination, but not the color congruity of the ground, since this does not belong to the depicted object. Appropriate testing of these alternatives involves contrasting performance across the color-congruent and color-incongruent conditions separately according to whether the manipulation of congruity applies to the figure or to the ground. Experiments 1 and 2 examined the impact of color con- gruity on short-term and long-term memory, respectively. In the case of long-term memory, the impact of color congruity was examined in two conditions. In one condition verbal recoding of the to-be-remembered stimuli was allowed, whereas in the other condition verbal recoding was prevented by articulatory suppression. According to the evidence reviewed above, the representation that supports image combination in short-term memory also supports performance in long-term memory when verbal recoding of the component stimuli is precluded. In this way, color congruity is expected to have comparable effects on short-term and long-term memory when, in the latter case, verbal recoding is prevented by articulatory suppression.

\section{EXPERIMENT 1}

\section{Method}

Subjects. One hundred sixty volunteers from 18 to 32 years of age took part in the experiment. They were all students at Lancaster University who were tested individually in their hall of residence. They were not paid for their participation.

Materials. Four sets of stimuli were prepared on cards measuring $17 \times 22 \mathrm{~cm}$. Each set consisted of six pairs of figures, the first figure in each case being a drawing of a nameable object. When combined, each pair of figures formed a composite figure that was itself easily named. The pictures were based on the line drawings used by Brandimonte et al. (1992b) and by Hitch et al. (1995). However, the color of an outline drawn figure can be ambiguous, being free to adopt either the color of the lines or the color of the background surface on which the lines are drawn. To remove this ambiguity and more clearly segregate the color of the figure from the color of the ground, the figures were produced as solid 2-D forms rather than as line drawings (Figure 1). Two sets of pictures were prepared for use in the conditions in which the color congruity of the figure was manipulated. One set consisted of black figures on a gray background, and the other set consisted of white figures on a gray background. Two sets of pictures were also prepared for use in the conditions in which the color congruity of the ground was manipulated. One set consisted of gray figures on a black background, and the other set consisted of the same gray figures on a white background. The alternative color formats for the to-be-combined pictures are illustrated in Figure 2 with reference to the manipulation of color congruity for one of the figures. Three additional pairs of combinable stimuli were drawn on transparencies for use in training and appeared as black figures on a white background.

Design. Twenty subjects were randomly assigned to each of eight experimental conditions, obtained by crossing the two factors stimulus region and color combination, with the constraint that the ratio of males to females was the same in each condition. Stimulus region refers to whether the color of the figure or the color of the ground of the to-be-combined stimuli was manipulated. Color combination refers to the colors assigned to the critical region of the to-be-combined pictures. The two values for color (black and white) yielded four combinations for the color of the first and second stimuli to be combined (white-white, black-black. whiteblack, and black-white). The first two of these four combinations represent the color-congruent conditions of the experiment, and the second two combinations represent the color-incongruent conditions. The presentation order of the stimuli was counterbalanced within each of the eight conditions, with half the subjects encountering the pairs in one random order and half encountering them in the reverse order. 

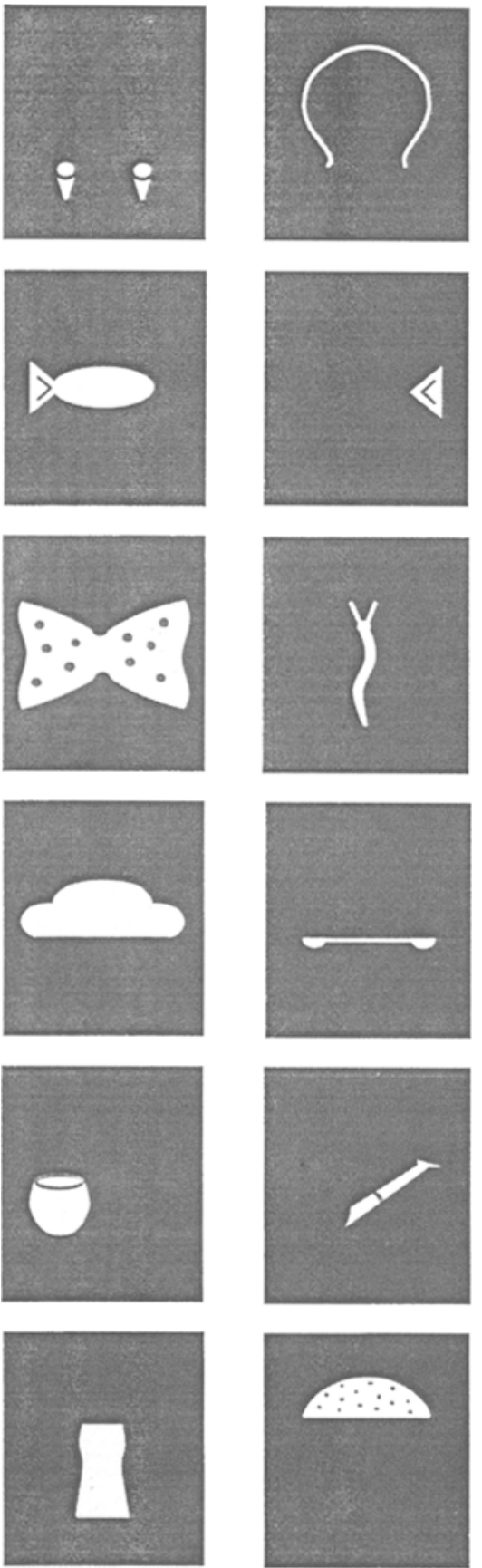

Figure 1. The six pairs of stimuli used in the image combination task, reproduced as white figures on a gray ground.

Procedure. Prior to the experimental trials, subjects received training in the image combination task using the transparencies The first stimulus of the first practice pair was presented for $2 \mathrm{sec}$, followed immediately by presentation of the corresponding second stimulus. The experimenter then superimposed the two transparencies by sliding one over the other to demonstrate how, when properly positioned, superimposition of the two patterns created a novel figure. The subject had to name the figure resulting from the combination. This procedure was carried out for each of the three pairs of practice stimuli.

Each subject was then shown the card containing the first member of the first pair of experimental stimuli for $2 \mathrm{sec}$. The card was then removed from view and replaced by the second member of the pair, again for $2 \mathrm{sec}$. Immediately after presentation of the second card, the subject was asked to combine the two stimuli in his/ her mind's eye and to identify the new figure resulting from this combination. This procedure was repeated for all six pairs of experimental stimuli.

\section{Results}

A response to the combination task was counted as correct if it was included among, or was an obvious synonym of, responses given by a separate group of subjects to pictures of the combined stimuli (see Brandimonte et al., 1992a; Hitch et al., 1995). Figure 3 shows the mean number of correct responses obtained in each condition, along with the corresponding confidence intervals, as
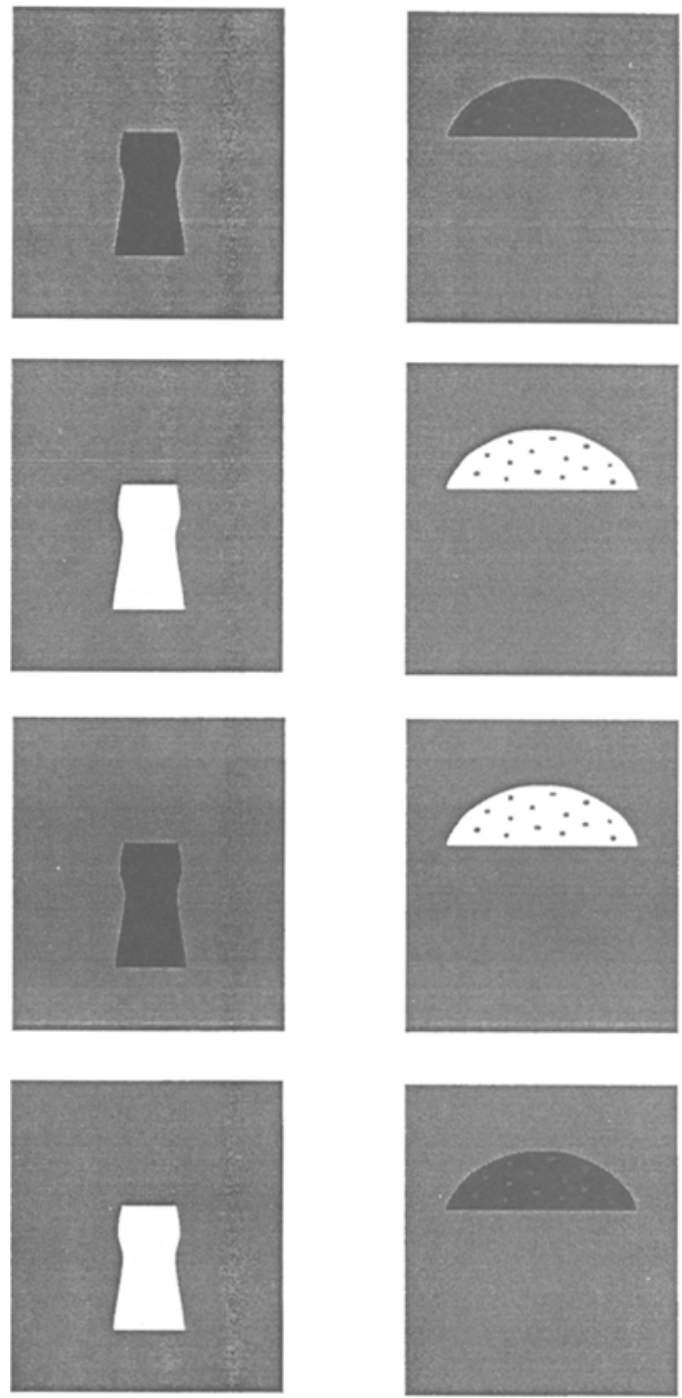

Figure 2. An illustration of the alternative color formats of the tobe-combined stimuli when the congruity of the figure is manipulated. 


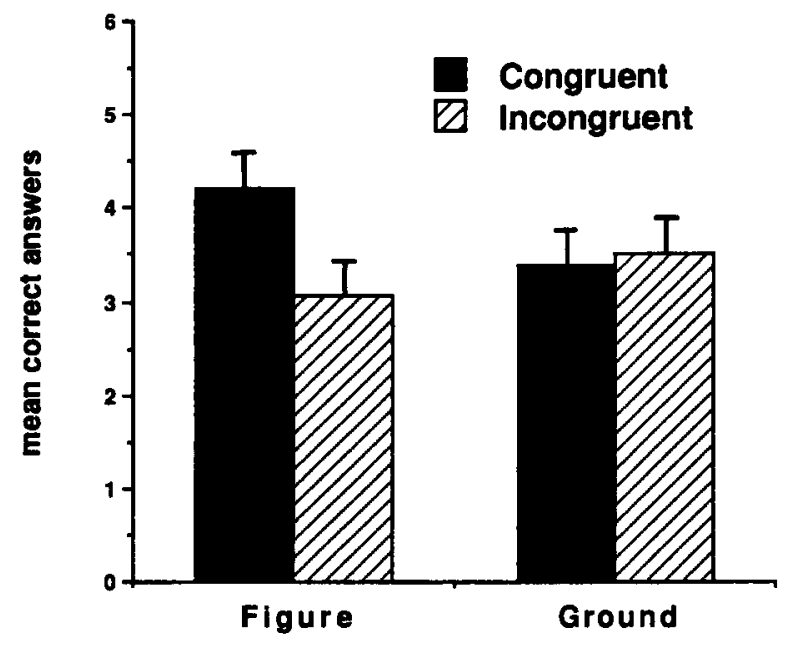

Figure 3. Results of Experiment 1. The mean number of correct image combinations (maximum $=6$ ) in short-term memory according to the color congruity of figure and ground.

recommended by Loftus and Masson (1994). It is clear that although image combination was impaired when the color of the figure was incongruent within each pair, it was not influenced by the color congruity of the ground.

\section{Discussion}

The results indicate that color incongruity impairs image combination when it involves the color of the figure but not when it involves the color of the ground. Because the visual representation associated with short-term memory does not appear to incorporate surface details of the ground against which an object is depicted, it does not seem to be a literal representation of the picture itself, but instead appears to be a representation of the depicted object. Although this representation is more abstract than a literal representation of the picture, the fact that it incorporates the depicted object's surface color confirms that it is less abstract than the view-independent visual representation normally associated with longterm memory (see Hitch et al., 1995).

\section{EXPERIMENT 2}

Hitch et al. (1995) have argued that the same visual representation that supports image combination in shortterm memory also supports performance in long-term memory when verbal recoding of the component stimuli is precluded. If so, then when verbal recoding is precluded, the differential effect of color congruity for figure and ground should also apply to long-term memory. Experiment 2 tested this prediction by repeating Experiment 1 using a long-term memory procedure. In one condition, verbal recoding was prevented by requiring subjects to suppress their articulation while endeavouring to learn the stimuli. In a second condition, no steps were taken to prevent verbal recoding, and, on the basis of previous results (Hitch et al., 1995), it was predicted that color congruity would have no effect on image combination performance.

\section{Method}

Subjects. Three hundred twenty subjects from 18 to 33 years of age were recruited in the same way as for Experiment 1 . They were all tested individually and were not paid for their participation. An equal number of subjects were assigned to the suppression and nosuppression conditions.

Materials and Design. Materials and design were the same as those in Experiment 1.

Procedure. Presentation of the experimental stimuli followed the long-term memory procedure described by Hitch et al. (1995). Subjects were shown the first members of the six pairs of stimuli and were required to learn these to the criterion that they were confident they could remember them in the order in which they

\section{LTM without suppression}

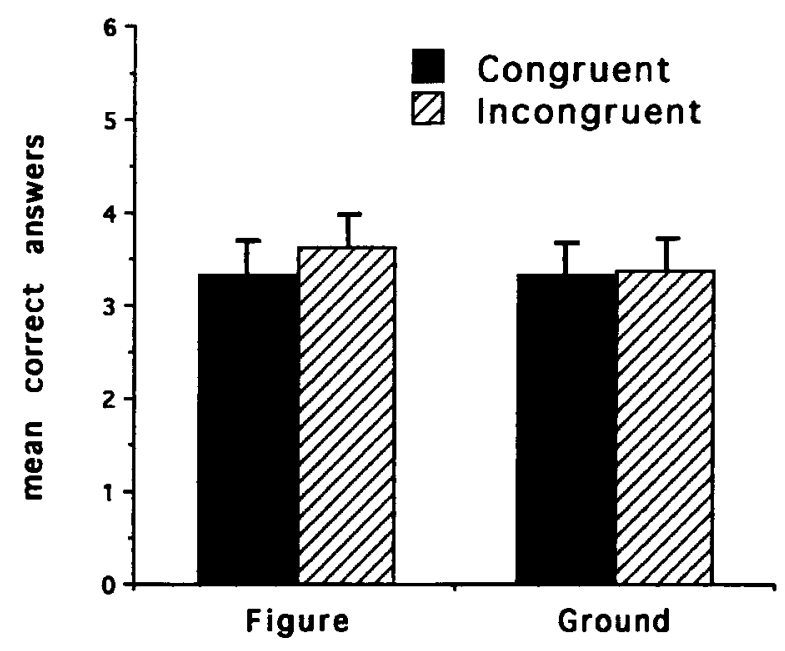

LTM with suppression

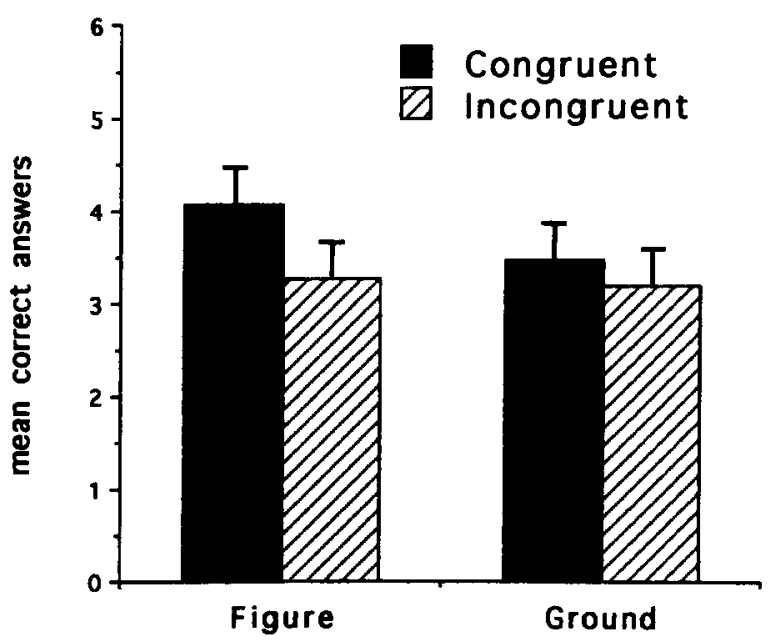

Figure 4. Results of Experiment 2. The mean number of correct image combinations (maximum $=6$ ) in long-term memory (LTM) according to the color congruity of figure and ground, shown separately for the conditions without (upper panel) and with (lower panel) articulatory suppression. 
had been learned. This was achieved by presenting the series of six pictures three times at a rate of $5 \mathrm{sec}$ per item (previous studies having shown this to be successful; see Brandimonte et al., 1992a, 1992c, and Hitch et al., 1995). Immediately after this learning phase, subjects received training in the image combination task in the manner described in Experiment 1. After practicing the task, they were asked to generate an image of the first picture of the learned series. They were then shown the other member of the pair for $2 \mathrm{sec}$ and asked to combine the two pictures mentally to discover the new form. Once they had named the resulting composite figure, the procedure was repeated until all six combinations had been attempted. Throughout the learning phase, half of the subjects were required to suppress their articulation by continuously uttering an irrelevant sound ("the-the-the ..."). Suppression was not required during the image combination phase. The other subjects were not required to suppress their articulation at any point.

\section{Results}

The criteria for successful image combination were the same as those used in Experiment 1. Figure 4 shows the mean number of correct responses in each condition of the image combination task, along with the corresponding confidence intervals. In the absence of articulatory suppression, it is clear that there were no effects of color congruity, whether this involved the figure or the ground of the to-be-combined stimuli. For the articulatory suppression condition, however, it is clear that image combination was impaired when the color of the figure was incongruent within each pair, but was not influenced by the color congruity of the ground.

\section{Discussion}

When verbal recoding is precluded, image combination based on long-term memory shows the same pattern of sensitivity to color congruity as was observed in the short-term memory condition of Experiment 1. Thus, color incongruity impaired image combination when it involved the color of the figure but not when it involved the color of the ground. Once again, therefore, image combination was being supported by a representation of the depicted object rather than by a literal representation of the picture itself. In this way, the results provide further support for the view that the form of visual representation supporting short-term memory also supports long-term memory when verbal recoding is precluded. When such recoding is allowed, image combination is insensitive to color congruity, confirming the involvement of a representation that does not preserve the surface characteristics (in this case color) of the to-be-combined stimuli.

Notice that the effect of articulatory suppression is being interpreted as arising from its preventing verbal recoding of the items rather than providing general dualtask interference. This interpretation is consistent with the known effects of item nameability on image manipulation (Brandimonte et al., 1992a, 1992c). Furthermore, if suppression had influenced performance simply as a secondary task making demands on central processing resources, it would have been expected to interfere with image combination rather than facilitate it (Fig- ure 4). Considering verbal recoding itself, this is not thought to reflect a shift toward relying on verbal memory codes to perform the image combination task, as such codes would not be adequate for that purpose. Instead, it is understood to reflect a shift toward relying on an alternative nonverbal representation that is particularly concerned with an object's structure rather than with its surface and view-specific features. Such a shift might reflect the dependence of object naming on the categorization of objects according to their structure, and it has already been noted in the introduction that object naming, in contrast with explicit picture recognition, draws on view-independent structural representations (see Biederman \& Cooper, 1992).

The present results may be related to the "verbal overshadowing" effect-the detrimental impact of generating a verbal description of a visual stimulus on recognition memory for that stimulus (Schooler \& EngstlerSchooler, 1990). This effect has been interpreted rather loosely as arising because verbalization produces a verbally biased memory representation that interferes with the application of the original visual memory. It is understood that the original visual memory remains uncontaminated by verbalization and is capable of supporting memory performance when, for example, recognition decisions are made under time pressure (Schooler \& EngstlerSchooler, 1990). It seems, however, that the alternative memory representation, though verbally biased, is not a verbal code. Thus, overshadowing occurs in relation to stimuli that are difficult to distinguish verbally, and the adequacy of the verbal descriptions generated by subjects shows no association with the accuracy of recognition in the overshadowing condition (Schooler \& EngstlerSchooler, 1990). By dissociating distinct forms of visual representation on the basis of their differential association with verbal recoding, the present study encourages the view that verbal overshadowing reflects the utilization of a nonverbal memory that is distinct from the original visual memory. In turn, the verbal overshadowing effect suggests that the differential association of verbal recoding with distinct forms of visual representation may not be confined to image manipulation.

\section{GENERAL DISCUSSION}

Previous studies have shown that two dissociable forms of visual representation support image combination. One form is relatively abstract, preserving an object's structure but not its surface color. It supports image combination when two conditions hold: when the procedure draws on long-term memory and when the stimuli are recoded verbally at encoding. If either of these conditions is not satisfied, image combination is supported by a more literal visual representation in which nonstructural information, such as surface color, is preserved.

The present results indicate that the representation preserving surface color is a description of the depicted object rather than a literal representation of the picture itself (i.e., it is not the pictorial code of Bruce \& Young, 
1986). Support for this comes from the finding that whereas the color congruity of the figure influences image combination, the congruity of the ground does not. Were it a literal representation of the picture itself, then the congruity of both figure and ground should have been influential.

There is other evidence that an object depicted in a picture, along with its nonstructural features, can be represented separately from the background against which it appears. For example, Wilton (1989) has shown that people can remember the color of a geometric figure very well despite, at the same time, struggling to remember the color of the background against which the figure appeared. ${ }^{1}$ In addition, Jolicoeur and Cavanagh (1992) have shown that the representation supporting the mental rotation of an alphanumeric character need not incorporate details of the background on which the character appeared. More specifically, they demonstrated that whereas the ability to rotate a mental image is sensitive to whether the character itself rotated during presentation, it is not sensitive to rotational movement of the background on which the character appeared.

It would seem, therefore, that at least three forms of representation can be derived from a picture of an object-a literal representation of the picture itself, a representation of the depicted object that incorporates nonstructural features, and a view-independent structural representation of the depicted object.

In isolating the second of these three representational forms, the present study has demonstrated comparable effects of color congruity on short-term and long-term memory, provided verbal recoding is prevented from contributing to performance based on long-term memory. This demonstration is consistent with evidence from previous studies of image combination indicating that the representation incorporating nonstructural information can contribute to image combination based on either short-term or long-term memory. In contrast, the contribution of the structural representation to image combination seems to be confined to long-term memory. As Hitch et al. (1995) pointed out, this evidence is incompatible with the view that the representations supporting short-term and long-term visual memory are respectively and exclusively associated with surface and structural descriptions (see Humphreys \& Bruce, 1989). Instead, the evidence from image combination is compatible with Kosslyn's (1980) view that whereas shortterm visual memory is concerned solely with the visual appearance of things, long-term visual memory is concerned with both the visual appearance of things and their structure. Thus, results from the image combination task converge with the conclusions drawn from recent studies of explicit picture recognition, object naming, and object decision discussed in the introduction.

\section{REFERENCES}

Avons, S. E., \& Phillips, W. A. (1980). Visualization and memorization as a function of display time and poststimulus processing time.
Journal of Experimental Psychology: Human Learning \& Memory, 6, 407-420.

Avons, S. E., \& PhILlips, W. A. (1987). Representation of matrix patterns in long- and short-term visual memory. Acta Psychologica, 65, 227-246

BIEDERMAN, I. (1987). Recognition by components: A theory of human image understanding. Psychological Review, 94, 115-145.

Biederman, I., \& CoOPER, E. E. (1991). Evidence for complete translational and reflectional invariance in visual object priming. Perception, 20, 585-593.

Biederman, I., \& CoOper, E. E. (1992). Size invariance in visual object priming. Journal of Experimental Psychology: Human Perception \& Performance, 18, 121-133.

Biederman, I., \& Gerhardstein, P. C. (1993). Recognizing depth-rotated objects: Evidence and conditions for $3 \mathrm{D}$ viewpoint invariance. Journal of Experimental Psychology: Human Perception \& Performance, 19, 1162-1182.

Brandimonte, M. A., Hitch, G. J., \& Bishop, D. V. M. (1992a). Influence of short-term memory codes on visual image processing: Evidence from image transformation tasks. Journal of Experimental Psychology: Learning, Memory, \& Cognition, 18, 157-165.

Brandimonte, M. A., Hitch, G. J., \& Bishop, D. V. M. (1992b) Manipulation of visual mental images in children and adults. Journal of Experimental Child Psychology, 53, 300-312.

Brandimonte, M. A., Hitch, G. J., \& Bishop, D. V. M. (1992c). Verbal recoding of visual stimuli impairs mental image transformations. Memory \& Cognition, 20, 449-455.

BRUCE, V., \& VALENTINE, T. (1985). Identity priming in the recognition of familiar faces. British Journal of Psychology, 76, 373-383.

BRUCE, V., \& YOUNG, A. W. (1986). Understanding face recognition British Journal of Psychology, 77, 305-327.

Christie, D. F. M., \& Phillips, W. A. (1979). Simple drawing and pattern completion techniques for studying visualization and long-term visual knowledge. Memory \& Cognition, 7, 360-367.

CoOper, L. A., \& SChaCter, D. L. (1992). Dissociations between structural and episodic representations of visual objects. Current Directions in Psychological Science, 1, 141-146.

Cooper, L. A., Schacter, D. L., Ballesteros, S., \& Moore, C. (1992). Priming and recognition of transformed three-dimensional objects: Effects of size and reflection. Journal of Experimental Psychology: Learning, Memory, \& Cognition, 18, 43-57.

Craik, F. I. M., \& LockharT, R. S. (1972). Levels of processing: A framework for memory research. Journal of Verbal Learning \& Verbal Behavior, 11, 671-684.

Ellis, R., Allport, D. A., Humphreys, G. W., \& Collis, J. (1989). Varieties of object constancy. Quarterly Journal of Experimental Psychology, 41A, 775-796.

FARAH, M. (1984). The neurological basis of mental imagery: A componential analysis. Cognition, 18, 241-269.

Hitch, G. J., BrandimonTe, M. A., \& WalKeR, P. (1995). Two types of representation in visual memory: Evidence from the effects of stimulus contrast on image combination. Memory \& Cognition, 23, 147-154.

Humphreys, G. W., \& Bruce, V. (1989). Visual cognition: Computational, experimental and neuropsychological perspectives. Hove, U.K.: Erlbaum.

Jolicouer, P., \& CaVAnagh, P. (1992). Mental rotation, physical rotation, and surface media. Journal of Experimental Psychology: Human Perception \& Performance, 18, 371-384.

KIKUCHI, T. (1987). Temporal characteristics of visual memory. Journal of Experimental Psychology: Human Perception \& Performance 13, 464-477.

Kosslyn, S. M. (1980). Image and mind. Cambridge, MA: Harvard University Press.

LofTus, G. R., \& Masson, M. E. J. (1994). Using confidence intervals in within-subject designs. Psychonomic Bulletin \& Review, 1, 476-490.

Phillips, W. A. (1983). Short-term visual memory. Philosophical Transactions of the Royal Society of London: Series B, 302, 295-309.

Phillips, W. A., \& Christie, D. F. M. (1977a). Components of visual memory. Quarterly Journal of Experimental Psychology, 29, 117-133.

Phillips, W. A., \& Christie, D. F. M. (1977b). Interference with visu-alization. Quarterly Journal of Experimental Psychology, 29, 637-650. 
ROBERTS, T., \& BRUCE, V. (1989). Repetition priming of face recognition in a serial choice reaction-time task. British Journal of Psychology, 80, 201-211.

Schacter, D. L., Cooper, L. A., \& Delaney, S. M. (1990). Implicit memory for unfamiliar objects depends on access to structural descriptions. Journal of Experimental Psychology: General, 119, 5-24.

Schacter, D. L., Cooper, L. A., Delaney, S. M., Peterson, M. A., \& Tharan, M. (1991). Implicit memory for possible and impossible objects: Constraints on the construction of structural descriptions. Journal of Experimental Psychology: Learning, Memory, \& Cognition, 17, 3-19.

SChOOLER, J. W., \& EngSTler-Schooler, T. Y. (1990). Verbal overshadowing of visual memories: Some things are better left unsaid. Cognitive Psychology, 22, 36-71.

WARREN, C., \& MORTON, J. (1982). The effects of priming on picture recognition. British Journal of Psychology, 73, 117-129.

WILTON, R. N. (1989). The structure of memory: Evidence concerning the recall of surface and background colour of shapes. Quarterly Journal of Experimental Psychology, 41 A, 579-598.

Young, A. W., HaY, D. C., McWeenY, K. H., Flude, B. M., \& Ellis, A. W. (1985). Matching familiar and unfamiliar faces on internal and external features. Perception, 14, 737-746.

\section{NOTE}

1. Wilton (1989) also demonstrated the same superior memory for the color of a figure over the color of the ground on which it appeared when the stimuli to be remembered were visual images generated from short verbal descriptions. This reinforces the general view, reflected in the present work, that perception and imagery share many resources and can draw on equivalent representational forms.

(Manuscript received May 9, 1995; revision accepted for publication March 26, 1996.)

\section{Nominations for the Editonship of Psychonomic Bulletin \& Review}

Nominations are solicited for the editorship of Psychonomic Bulletin \& Review. The term of the present editor, Henry L. Roediger III, expires at the end of 1998. The new editor will begin an official 4- or 5year term on January 1, 1999, and will begin to receive manuscripts early in January 1998. The Publications Committee of the Psychonomic Society expects to appoint the new editor by December 1997.

Nominations (including self-nominations) should be submitted by August 31, 1997, to:

Geoffrey Loftus

Chair, Psychonomic Bulletin \& Review Search Committee

Department of Psychology

University of Washington

Seattle, WA 98195 